\title{
TRATADOS DE LIBRE COMERCIO Y LOS IMPACTOS ECONÓMICOS - SOCIALES
}

Julio López Mas ${ }^{(*)}$

E-mail: johans_jlopez@yahoo.com

\begin{abstract}
RESUMEN
El presente artículo intenta describir y explicar los principales impactos económicos y sociales distinguiendo la estructura económica del Perú, en su carácter de economía primaria exportadora, la gran y mediana empresa como la pequeña empresa, a quienes el impacto de medidas arancelarias afecta en cuando menos dos aspectos. Se discuten diferentes puntos de vista en los temas prioritarios de agenda para que los beneficios de éste y otros acuerdos comerciales, no sean absorbidos por una fracción pequeña de la población, sino por la mayoría.
\end{abstract}

Palabras Clave: Tratados de Libre Comercio, empleo, impactos sociales, estructura económica del Perú.

\begin{abstract}
The current article tries to describe and to explain the main social and economic impacts distinguishing the peruvian economic structure, in its character of exporting primary economy, the great and medium business as the small business, to those whom the impact of tariff measures affects in at least two aspects. Different points of view in the high-priority themes of agenda are discussed so that the benefits of these and other trade agreements are not absorbed for a fraction of the population, but by the majority.
\end{abstract}

Key Words: Free Trade Agreement, employment, social impacts, economic structure of Perú.

(*) Doctor en Economía e Historia Latinoamericana. Licenciado en Economía Política. Profesor Principal de la Facultad de Ciencias Administrativas (UNMSM). Ha sido miembro de la Secretaría Técnica para el Proceso de Descentralización del Ministerio de la Presidencia y ex Asesor de la Dirección Ejecutiva de FONCODES; actual Jefe de la Oficina de Calidad Académica y Acreditación de la Facultad. 


\section{INTRODUCCION}

En el presente artículo se señalan algunas condiciones y comportamientos estructurales e históricos de la economía peruana desde las reformas de los años noventa hasta nuestros días, así como el origen y formulación del Acuerdo de Preferencias Arancelarias (ATPA-ATPDEA).

A continuación se describen algunos aspectos de importancia que son característicos de la economía peruana y que tienen relación con el acuerdo. En particular se presentan tres aspectos que son fuente de posibles conflictos entre las expectativas de la población y los reales efectos del acuerdo, los cuales son el empleo, estructura productiva y la informalidad.

Posteriormente se analizan algunas posturas frente al TLC desde diversos puntos de vista, discrepantes muchos, pero todos ellos coincidentes en la necesidad de enfocar la atención a los posibles conflictos sociales, como barreras institucionales, productividad, compensaciones, etc., además de proporcionar las capacidades necesarias que el acuerdo demanda.
Por último se plantean para el debate posterior algunas observaciones que, como resultado de los futuros acontecimientos, nos llevan a la reflexión sobre la necesidad de atender a los problemas estructurales del país antes de enfocar la atención en el acuerdo mismo, ya que la prioridad es acercar el acuerdo a los que estructuralmente no pueden acceder a él, como son los agricultores de las zonas más alejadas y con economías de subsistencia.

\section{ANTECEDENTES}

La apertura comercial en el Perú es un fenómeno que se lleva a cabo desde principios de los años 90. Anterior a ello, alternativas como la sustitución de importaciones, propia de los años 60 , o la regulación de los años 80 , eran características del comercio exterior peruano.

El Gráfico 1 presenta el nivel de arancel promedio desde 1990 hasta nuestros días. En el Gráfico 2 se puede observar que coincide la reducción del arancel promedio con el crecimiento del Producto Bruto Interno. Además el ingre-

\section{Gráfico 1}

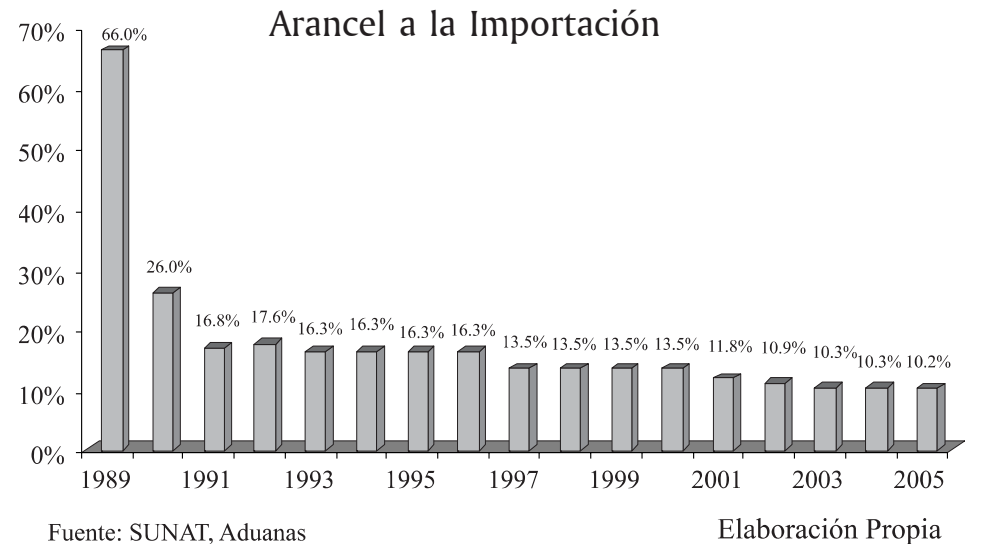

Gráfico 2

PBI: Variaciones Porcentuales

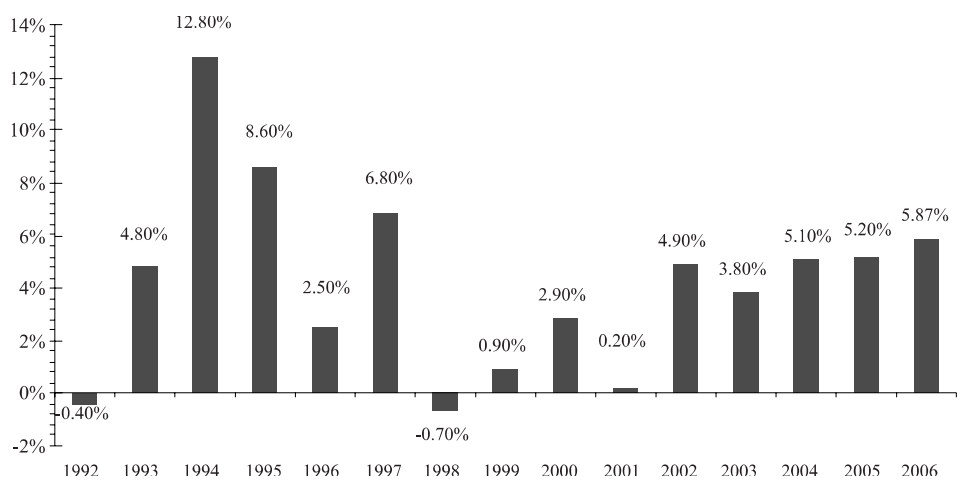

Fuente: BCRP. Elaboración Propia. 
so nacional también ha aumentado considerablemente, sin embargo es en los últimos cinco años en donde la diferencia entre producto bruto y ingreso nacional se ha reducido.

Como vemos en el Gráfico 3 el Producto Nacional Bruto (PNB), que muestra el producto agregado de los residentes en el territorio nacional, representa una disminución con respecto al valor tendencial frente al ingreso nacional disponible, que considera además las transferencias corrientes (como por ejemplo remesas, etc.)

Gráfico 3

PBI, PNB e YND (1996-2005)

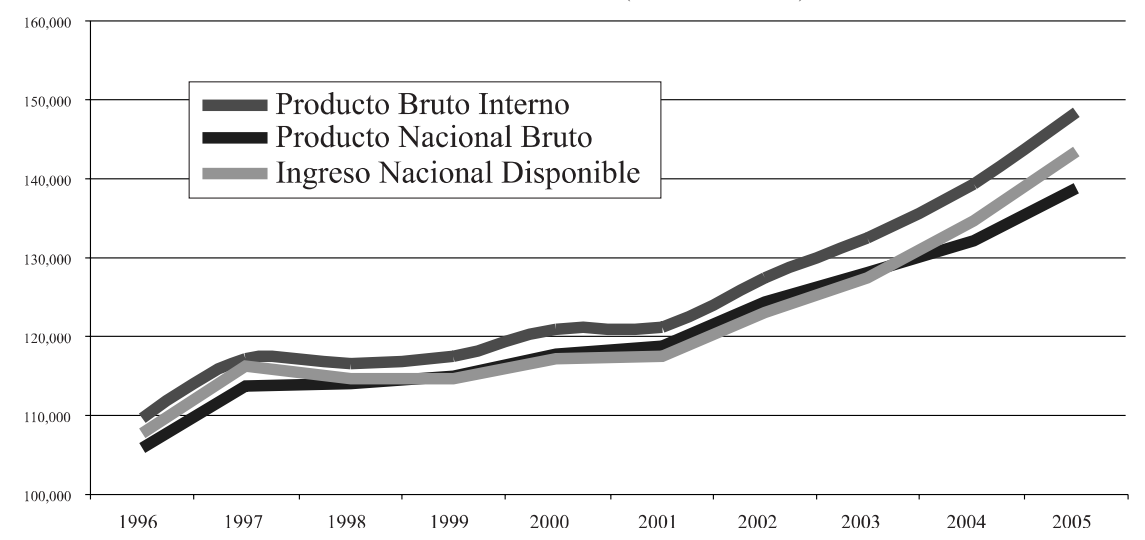

Fuente: BCRP. Elaboración Propia.

El Gráfico 4 nos muestra otro aspecto de este fenómeno como son los términos de intercambio, es decir, la relación entre el valor de lo exportado y lo importado cuya tendencia ha variado sustantivamente en cuanto a sus características durante los últimos seis años, pasan- do de una economía que privilegiaba la importación a una que promueve la exportación.

Como vemos en ambos escenarios, la visión de interrelación con el mundo se hace patente: es necesario comerciar con el mundo.

\section{Gráfico 4}

Términos de Intercambio $(1994=100)$

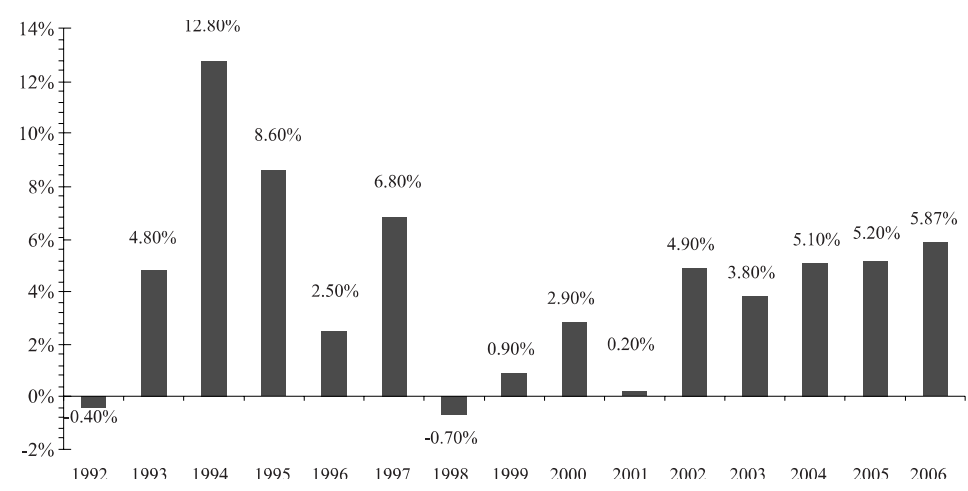

Fuente: BCRP. Elaboración Propia.

A nivel interno existen graves desfases entre las cadenas de producción, consumo, ingreso y distribución de la riqueza, cuyo resultado es que mientras más riqueza existe en el Perú, esta se encuentra más concentrada o desigualmente distribuida, y no solamente por la existencia de fallos de mercado tales como monopolios naturales, externalidades o indefinición de los derechos de propiedad, sino por la existencia de una estructura económica particular que se caracteriza por un alto grado de informalidad y de incentivos para incumplir con la ley.

De Soto ${ }^{1}$ refiere algunos costos, por ejemplo el costos de ser ilegales que se manifiesta en: 
a) Evitar la sanción.

b)Transferencias netas entre informales y formales, en perjuicio de los primeros.

También los costos de no tener una buena ley:

a)Por falta de derechos de propiedad.

b)Por no aprovechar el sistema contractual.

c) Por la ineficiencia del derecho extracontractual (característico de la informalidad).

Por ejemplo, las sucesivas normas de flexibilización laboral, en vez de promover un empleo que promueva la competitividad en el mercado de trabajo y por ende, proporcione recursos humanos de mejor calidad, ha provocado exactamente el efecto contrario: la desnaturalización del mercado laboral, que se ha servido de las distintas formas contractuales para ocultar la existencia del vínculo y, por consiguiente, el cumplimiento de las obligaciones sociales.

Se dice que hemos transitado de un extremo a otro del péndulo del tratamiento económico de la sociedad. Desde una política comercial restrictiva, que impidió sistemáticamente la importación de algunos bienes entre los que se encuentran los bienes de capital y que a la larga determinó el desfase tecnológico de la segunda etapa; a la apertura comercial indiscriminada, que desprotegió la industria consolidada y a la industria nueva. El perjuicio inmediato se observó en el empleo, el ingreso y el consumo.

Algunas condiciones políticas predominantes hicieron que no ocurrieran desembalses sociales que hubieran caracterizado un periodo de esta naturaleza; se amplió la cobertura de algunos servicios de seguridad social, y el déficit del sector público se incrementó proporcionalmente a la reducción de las restricciones comerciales. El gobierno de ese entonces (1990-2000) se preocupó por ocultar los problemas estructurales de la sociedad, y la informalidad ganó terreno en todos los sectores.

La gestión del gobierno del período 20012006 no ha significado, sin embargo, la restitución de la normalidad en el sistema económico, que equipare la producción, el ingreso, el consumo y la distribución de la riqueza; muy por el contrario, el Perú nuevamente se ha quedado a la zaga en la transformación de su estructura productiva, retornando a la actividad minera y pesquera como las predominantemente generadoras de ingreso en el Perú. Más adelante se observará la estructura de las exportaciones peruanas en el Gráfico 7.

En ese contexto y durante este periodo, la política exterior peruana se preocupó por renovar las relaciones con sus socios comerciales, ofreciendo por una parte facilidades para la inversión extranjera y por otra parte, el establecimiento de acuerdos comerciales y tratados de libre comercio, como por ejemplo los firmados con Tailandia, Singapur, Chile, y el más discutido de todos, con Estados Unidos.

La Ley de Protección Comercial y Erradicación de la Droga (ATPDEA por sus siglas en inglés) es el principal antecedente de política comercial entre el Perú y Estados Unidos. Fue promulgada por el actual presidente del país del norte en agosto del 2002, y constituye un régimen de excepción del pago de aranceles otorgados de forma unilateral a Bolivia, Colombia, Ecuador y Perú, constituyéndose en una manera de renovar los beneficios de la Ley de Preferencias Arancelarias Andinas (ATPA por sus siglas en inglés), establecida el 4 de diciembre de 1991, aunque recién se hizo efectiva en el Perú en 1993, con vigencia hasta el 4 de diciembre del 2001.

Según un artículo de Roberto Abusada, el ATPA/ATPDEA ha permitido superar ciertas trabas a la competitividad que eran exógenas a las empresas, tales como el déficit de infraestructura de transporte, elevados costos laborales, limitado acceso al crédito, entre otros, al permitir la entrada sin aranceles al mercado norteamericano de los productos involucrados en el sistema. Su contribución al PBI alcanza el $3.3 \%$ (en el año 2000), y a pesar de que las exportaciones de derivados del cobre dominaban la canasta de productos bajo preferencias, el grueso de las personas ocupadas laboran en actividades del sector agropecuario.

«...en relación al impacto total, se pasó de 5,7 miles en 1993 a 287 mil personas ocupadas en actividades relacionadas a la exportación, en el 2001 bajo ATPA/ATPDEA. Cabe mencionar que Lima ha sido la región que más se ha beneficiado²».

El TLC con los Estados Unidos se ha constituido en una muestra de la forma en que los grupos involucrados se han puesto de acuerdo, y del predominio de una de las posturas, que a pesar de eso cuenta con poco apoyo de la po- 
blación. Está documentado que la aprobación al TLC cuenta con una aprobación del $45 \%^{3}$, principalmente en zonas urbanas de la costa, donde el impacto de las actividades que están directamente relacionadas con el comercio exterior con Estados Unidos es mayor y está correlacionada con el nivel de ingresos y, por consiguiente, con el consumo y el bienestar.

En zonas como la sierra central y sur, además de la selva peruana, la aprobación por el TLC es baja porque se entiende como una política expansionista y de destrucción de la industria nacional por la incorporación de productos norteamericanos, lo que no significa que sean las únicas poblaciones del Perú que se verán afectadas por las incidencias del acuerdo comercial. Para bien o para mal son muchas las actividades que serán afectadas por el acuerdo, y para compensar a los grupos de interés más sensibles (en particular, los agricultures tradicionales), el Estado ha establecido algunos mecanismos.

El impacto del TLC en el Perú es probablemente mayor porque la estructura socio-económica de nuestro país es diferente de la estructura del socio comercial, y son muchas las actividades que necesitan un tiempo para empezar a ser competitivas. En las siguientes líneas se intentará explicar algunos de los impactos en tres aspectos: la estructura productiva, informalidad y empleo.

\section{ESTRUCTURA PRODUCTIVA DEL PERÚ}

El Perú está caracterizado por una serie de aspectos entre los que se encuentran:
- Empleo insuficiente y de baja calidad.

- Predominancia de actividades primario exportadoras.

- Informalidad

Estos elementos influyen de forma determinante en el impacto de los acuerdos de apertura comercial, y justifican argumentos tales como que el 2\% de las empresas en el Perú aprovecharán los beneficios del acuerdo. Aunque ciertamente el Perú no representa un gran mercado para los bienes de consumo directo para los Estados Unidos, sí es atractivo para el esquema norteamericano de promoción comercial, para mantener su ámbito de influencia en América Latina, fortalecer lazos políticos y acentuar su predominio en actividades tales como la propiedad intelectual y alta tecnología.

Estos tres aspectos mencionados líneas arriba impiden que las empresas peruanas se incorporen en cadenas exportadoras.

\section{Empleo}

En el Perú un 45.2\% de la PEA (al 2005) se encuentra empleado y durante el mismo periodo el $46.7 \%$ se encuentra sub empleado. Las estadísticas del Instituto Nacional de Estadística e Informática (INEI) definen sub empleo en función de dos aspectos: el trabajador percibe ingresos menores de S/. 1,100 (el monto de la canasta básica familiar para el periodo 2004) o la persona trabaja más de 50 horas semanales para obtener dicho ingreso mínimo. Veamos el siguiente cuadro:

Cuadro 1

Perú: Empleo Urbano (1997-2005) (en porcentajes)

\begin{tabular}{|l|r|r|r|r|r|r|r|r|r|}
\hline \multicolumn{1}{|c|}{ Nivel de Empleo } & 1997 & 1998 & 1999 & 2000 & 2001 & 2002 & 2003 & 2004 & 2005 \\
\hline Total & 100.0 & 100.0 & 100.0 & 100.0 & 100.0 & 100.0 & 100.0 & 100.0 & 100.0 \\
\hline Empleado & 47.2 & 48.3 & 48.8 & 50.0 & 44.5 & 47.6 & 46.3 & 46.9 & 45.2 \\
\hline Subempleado & 45.0 & 43.9 & 43.2 & 43.1 & 47.6 & 42.7 & 42.9 & 42.8 & 46.7 \\
\hline Visible (horas) & 17.0 & 14.6 & 13.6 & 13.3 & 14. & 11.7 & 9.8 & 12.6 & 10.7 \\
\hline Invisible (ingresos) & 28.1 & 29.4 & 29.6 & 29.8 & 33.1 & 31.0 & 33.1 & 30.2 & 36.0 \\
\hline Desempleo & 7.7 & 7.8 & 8.0 & 7.4 & 7.9 & 9.7 & 10.8 & 10.3 & 8.1 \\
\hline
\end{tabular}

Fuente:SNE Ministerio de Trabajo y Promoción del Empleo (MTPE). Encuesta Nacional de Hogares Niveles deEmpleo. Elaboración Propia. 
Según lo observado, la tasa de creación de nuevos empleos es menor a la población adecuadamente empleada, (alrededor de 1\%), mientras que en el sub empleo se encuentran las mayores tasas de creación de nuevos empleos. En particular, el sub empleo invisible, que es aquel que se determina por una menor capacidad del empleado de obtener ingresos a partir de una única fuente de empleo, por lo que se ve obligado a emplearse en más de una actividad para poder completar su canasta de consumo.

\section{Gráfico 5}

Estructura del Empleo: Lima Metropolitana

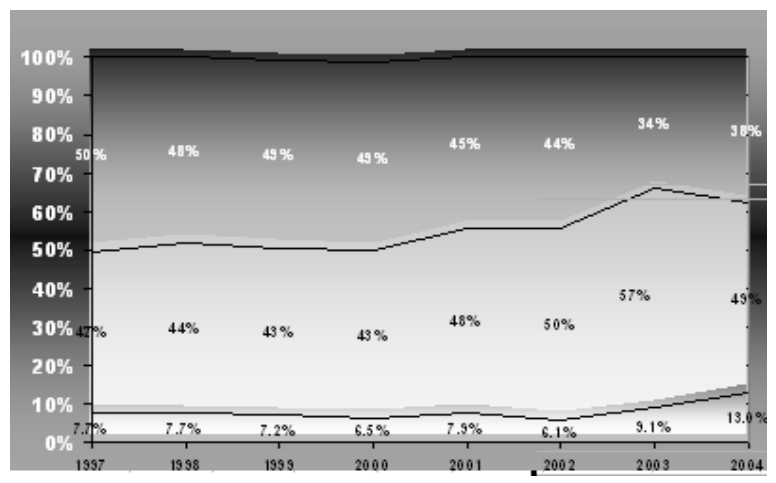

Es posible que el trabajador peruano (en actividades como minería, pesca, telecomunicaciones, etc.) tenga la misma productividad que un trabajador de otras zonas del mundo, como Estados Unidos, dado el alto grado de inversión en tecnología y la capacitación que las empresas generan para este empleo. Sin embargo en otras actividades tales como agricultura, industria intermedia (acero, refinación, etc.), o la industria de bienes de consumo (alimentos, textil, etc.), la productividad media del trabajador peruano es mucho menor. Esto considerando las actividades en que se emplea mano de obra for-

\section{Gráfico 6}

Estructura del Empleo: Perú Urbano

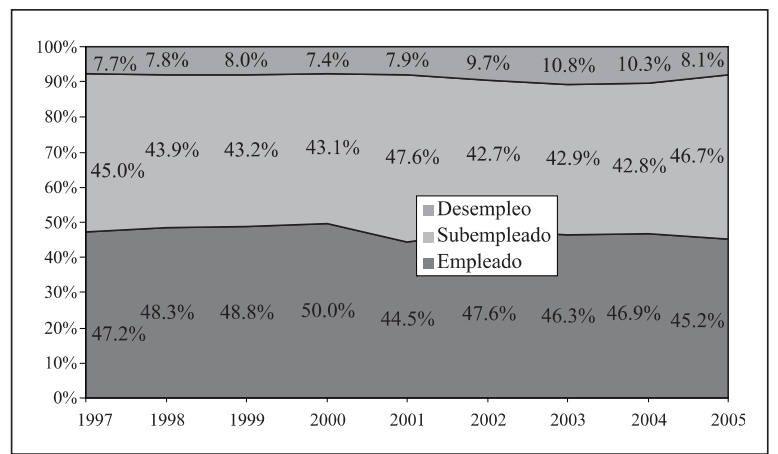

Fuente: ENAHO- INEI - 2005. Elaboración Propia. malmente, puesto que en otras actividades como servicios (transporte, por ejemplo), la productividad es muy baja. Hay una correspondencia entre inversión y empleo. Es curioso el hecho que muchos peruanos que están en el extranjero son más productivos en promedio que sus pares dentro del país, debido a que aquí no se cuenta con los mismos niveles de capital / trabajo que en otros países, donde la productividad de la mano de obra es mayor ante la presencia de mejores condiciones de trabajo, mayor tecnificación e instrumentos para realizar las tareas, además de las mayores remuneraciones.

Durante los años noventa, el Estado absorbía un aproximado del $22 \%$ de la PEA en la forma de programas sociales, burocracia, etc. Posteriormente se ha llegado a un $15 \%$ del total, pero debe considerarse que una gran parte del empleo que absorbe el sector público en la actualidad no es productivo, en el sentido que no genera la producción de bienes y servicios, sino que se destina al sostenimiento del Estado.

Por otra parte la intermediación ha sido una nueva fuente de empleo. El comercio, por ejemplo, ha extendido la cadena de distribución en algunas actividades como agricultura, pesca, silvicultura, etc., que llegan a los mercados tras haber pasado por muchas personas que generan sus ingresos con el diferencial de precios; es así que vemos que los productores primarios perciben muy poco y el consumidor final asume precios altos, que en gran medida no se justifican con el valor agregado proporcionado por la cadena de distribución.

El empleo formal en el Perú también está caracterizado por la existencia de mayores costos con respecto al empleo no formal, por la existencia de rubros considerados por los empresarios del país como sobrecostos como el pago de CTS, vacaciones, compensaciones por despido arbitrario, etc., y que en el entender de los empresarios, impiden una adecuada flexibilización del mercado laboral y por lo tanto de su formalización. Por el contrario, los sindicatos manifiestan que es precisamente el empresariado el que ha provocado una informalización del empleo, haciendo caso omiso de las obligaciones laborales o mal utilizando otras modalidades de contratación como los servicios no personales, locación de servicios, formación laboral juvenil, etc. Lo irónico es que el Estado también es juez y parte en este uso indiscriminado de 
los contratos laborales. Por ese motivo el problema del empleo genera constantes conflictos entre grupos de interés aparentemente antagónicos, especialmente ante la eventualidad de un cambio de los patrones de comercio, gracias a los acuerdos comerciales como el TLC.

En un extenso artículo estadístico, los consultores Seminario y Molina refieren que el efecto directo sobre el empleo a partir del incremento de las exportaciones es de 32,552 puestos de trabajo (alrededor 1\% de la PEA ocupada), y este resultado disminuye considerablemente si los sectores primarios no responden, por lo que conviene promover los encadenamientos internos en nuestra economía. El efecto total de las exportaciones sobre el empleo representa 85,467 puestos de trabajo (cerca del $1.7 \%$ de la PEA), mientras que el empleo neto es de 18,148 puestos de trabajo ${ }^{4}$

Como vemos el efecto del TLC, con todo lo ofrecido por los medios de comunicación, aún resulta insuficiente como alternativa de generación de nuevas fuentes de trabajo. La firma del acuerdo por sí sola, sin entender que el crecimiento del empleo está también constituido por otras dinámicas -ligadas a aspectos de demanda interna, productividad y diseño de políticas públicas- no cambiará la realidad existente.

\section{Actividades Primario - Exportadoras}

Una misma actividad económica tiene dos comportamientos para el análisis de cuentas nacionales:

- Funcionales: Las unidades económicas se comportan como establecimientos que se aglutinan en torno a un proceso productivo. Producen bienes y servicios y generan ingresos.

- Institucionales: las empresas constituidas formalmente que además de tener un proceso productivo o más de uno, poseen contabilidad completa. De ellas, INEI obtiene información estadística referida a la producción e ingreso.

En el Cuadro 2 observamos que las actividades económicas en el Perú están organizadas por sectores económicos que generan, al fin y al cabo, bienes y servicios para la economía. El sector público, a pesar que es un componente importante de la economía, se le considera como complementario al sistema económico.
Cuadro 2

Unidades y Sectores Económicos

\begin{tabular}{|c|c|c|}
\hline \multicolumn{2}{|c|}{$\begin{array}{l}\text { Unidades } \\
\text { Funcionales }\end{array}$} & $\begin{array}{c}\text { Unidades } \\
\text { Institucionales }\end{array}$ \\
\hline Extracción & Actividad Primaria & \multirow{5}{*}{$\begin{array}{c}\text { EMPRESA } \\
\text { Petroperú } \\
\text { Repsol } \\
\text { Grifos Shell }\end{array}$} \\
\hline Refinación & Actividad Secundaria & \\
\hline Distribución & Actividad Terciaria & \\
\hline Venta al menudeo & Actividad Terciaria & \\
\hline \multicolumn{2}{|l|}{ GASOLINA } & \\
\hline Funte: ENAHO - INEI & Elaboración : & \\
\hline
\end{tabular}

En el Perú la predominancia de las actividades primarias a lo largo de la vida republicana es determinante de su estructura económica (actividades mineras, agrícolas, pesca, forestal, etc.), sin que a diferencia de otros países, esta abundancia de materias primas esté interrelacionada con una industria que las procese y que las convierta en bienes y servicios competitivos. Es esta predominancia la que, en cierta forma, impide que se homogenicen los resultados del beneficio económico.

El Gráfico 7 muestra la evolución y composición de las exportaciones peruanas. Nótese en las líneas punteadas aquellos productos tradicionales, que representan más del 60\% (alrededor de US\$ 12,300 millones), provenientes de la explotación de materias primas.

\section{Gráfico 7}

Estructura de las Exportaciones

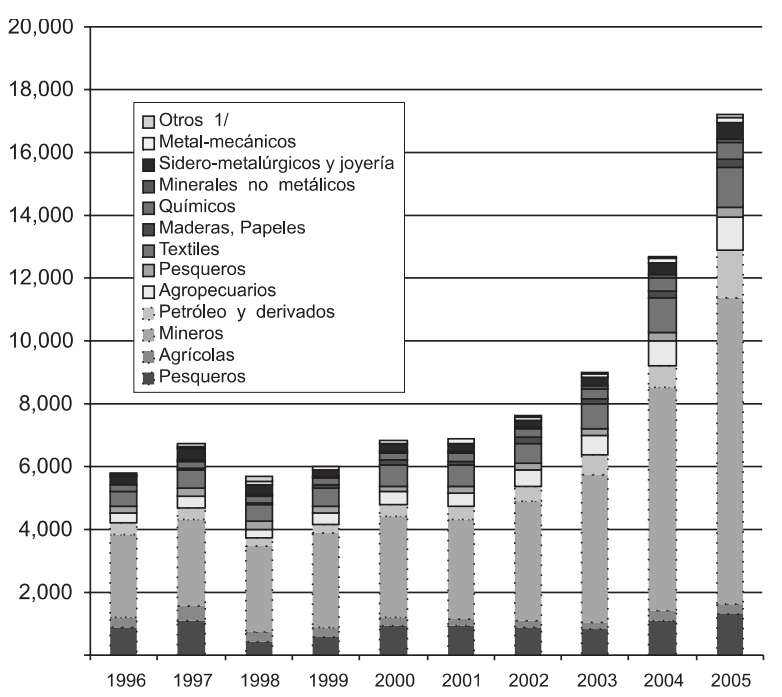

Fuente: BCRP. Elaboración Propia.

Sobre esto el estructuralismo latinoamericano, comandado desde CEPAL, pone de manifiesto que en la interrelación entre los países centrales y los países periféricos, en un sistema que 
Prebisch denomina Sistema Centro Periferia, encontramos las siguientes características:

a.Estructuras productivas de los países centrales homogéneas, mientras que en los periféricos son heterogéneas. Por ejemplo, la coexistencia (en los países periféricos) de agricultura de alta productividad con agricultura de subsistencia.

b.Estructuras económicas especializadas, en donde actividades muy específicas generan economías de escala, altos niveles de productividad y complementariedad a través de integración vertical y/u horizontal, y presencia de enclaves con poco efecto de arrastre.

c.Se revela ante la existencia de estas economías especializadas la manifiesta división internacional del trabajo, para la cual los países periféricos están conectados a los centrales en la provisión de algún grupo de actividades económicas.

d.Las relaciones entre centro y periferia son asimétricas, por tanto el libre comercio no corrige ni reduce las desigualdades internacionales sino que en cierta medida las acentúa ${ }^{5}$.

Aunado al hecho de una estructura productiva heterogénea y especializada en pocas actividades económicas (siguiendo los términos del estructuralismo), en el Perú se conjugan un Estado ineficiente, un sistema tributario complicado y un esquema laboral con muchas inequidades, así como la dependencia que nuestra economía tiene respecto de la moneda extranjera, tanto en nuestras actividades de comercio como en el ciclo de ahorro-inversión.

En un artículo anterior mencionaba que el sistema económico regional peruano se basaba en cuatro factores:

- La geografía.

- Las tendencias históricas de organización y utilización del espacio.

- El modelo económico.

- Las políticas públicas.

A la vez refería que las regiones se han desarrollado de manera desigual, siguiendo el patrón de crecimiento primario exportador, semi industrial y dependiente, en palabras de Efraín González, quien sostiene que el principal efecto del programa de ajuste ha sido el incremento de la centralización en Lima, "neo-centralismo", fundado en la primarización de la economía y el distanciamiento del centro con los periféricos. En conclusión, las reformas de ajuste de los noventa no han concluido en un desarrollo homogéneo, sino en relaciones bastante heterogéneas entre el centro y la periferia regional.

Distinguía en dicho artículo, además, cuatro tipos de regiones:

- Central, en que se encuentran todos los mercados de bienes, servicios, laboral y capitales y que absorbe casi la mitad del PBI.

- Descentralizadas, donde existen mercados de bienes y laboral.

- De colonización, similares a las descentralizadas, pero con una escasa participación en el PBI.

- Espacios mercantiles, con mercados de bienes y débiles mercados de trabajo, con gran componente de economías de subsistencia y baja participación del PBI, que además concentra a la población rural ${ }^{6}$.

\section{Informalidad}

En el Gráfico 8 vemos en el porcentaje del PBI la evolución de la Presión Tributaria (2006 y 2007 estimados). Es notable el hecho que durante el periodo de liberalización de la economía entre 1996 y 1999 es cuando más presión tributaria ha habido, y que corresponde al periodo de mayor "estabilidad" política; lo contrario sucede entre el periodo entre 1999 y 2002.

Sin embargo hay una relación inversa entre la formalidad y presión tributaria, porque al Estado se le hace muy sencillo cobrar impuestos a empresas grandes, ya constituidas y cuyo costo de cobranza es relativamente menor que la pequeña y mediana empresa. El Estado no se ha preocupado por formalizar a las empresas, ni promover los beneficios de la formalidad. Hernando de Soto manifiesta que la legalidad impone costos que son mayores que los beneficios de ésta y que los costos de la informalidad, al ser menores, incentivan a las unidades económicas a alejarse de la formalidad. En países como el Perú, la inversión informal (en infraestructura de vivienda, transporte y comercio) supera a la inversión estatal en los mismos rubros, según su investigación. A su vez, lo que deno- 
minamos como formalidad está dentro de una "campana de vidrio" a la que no accede la mayoría de los ciudadanos.

En el Perú existen alrededor de 2 millones de empresas que forman parte del empresariado nacional, pero sólo el 2\% de ellas pueden acceder apropiadamente a la formalidad (que se entiende, en este caso, por la constitución de la empresa, el pago de tributos, cumplimiento de obligaciones laborales, licencias y autorizaciones de operación, etc.).

Como se observa en el Cuadro 3, la empresa formal representa además el $97 \%$ de las exportaciones, y ocupa al $10 \%$ de la PEA ocupada a nivel nacional, mientras que en su conjunto la empresa informal representa cerca del $80 \%$ número de empresas y emplea al 68\% de la PEA, sin participación directa en el TLC. Aquí encontramos a la pequeña y micro empresa, que podría alimentar dentro del país a la cadena exportadora, pero que su acceso directo al comercio exterior es limitado.

En este sentido, lo resaltante no es la confrontación entre los que acceden y los que no acceden sino que las medidas que el Estado tome para favorecer el TLC deben a su vez considerar que hay una gran proporción de empresas para las que es estructuralmente imposible obtener beneficios, pero sí la imposición de costos, como por ejemplo una modificación del mercado laboral al aumentar la oferta de empleados calificados sin empleo, que habitualmente trabajan en actividades cuya producción es sustituida por la importación libre de aranceles proveniente de los Estados Unidos.

Gráfico 8

Ingresos Tributarios y Presión (\% del PBI)

\begin{tabular}{|c|c|c|c|c|c|c|c|}
\hline & & \multicolumn{2}{|c|}{ Cantidad } & \multicolumn{2}{|c|}{ Empleo } & \multicolumn{2}{|c|}{ Exportaciones } \\
\hline & & $\begin{array}{c}\mathrm{N}^{0} \text { de } \\
\text { empresas }\end{array}$ & $\%$ & $\begin{array}{c}\mathrm{N}^{0} \text { de } \\
\text { empleados }\end{array}$ & $\begin{array}{c}\% \text { de la PEA } \\
\text { ocupada }\end{array}$ & $\begin{array}{l}\text { Valor (US\$ } \\
\text { millones) }\end{array}$ & $\%$ \\
\hline $\begin{array}{c}\text { Empresas con } \\
\text { instituciones } \\
\text { para crear } \\
\text { capital }\end{array}$ & $\begin{array}{l}\bar{\pi} \\
\stackrel{5}{5} \\
0 \\
\leftarrow\end{array}$ & 52133 & $2 \%$ & 626,341 & $10 \%$ & 16,382 & $97 \%$ \\
\hline \multirow{2}{*}{$\begin{array}{l}\text { Empresas sin } \\
\text { instituciones } \\
\text { para crear } \\
\text { capital }\end{array}$} & $\begin{array}{l}\bar{\sigma} \\
\text { है } \\
\text { ठ } \\
\stackrel{L}{0}\end{array}$ & 439,253 & $17 \%$ & 1370,945 & $22 \%$ & 507 & $3 \%$ \\
\hline & 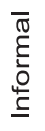 & 2121,791 & $81 \%$ & 4322,234 & $68 \%$ & $0 \%$ & $0 \%$ \\
\hline \multicolumn{2}{|l|}{ Total } & 2613,177 & $100 \%$ & 6319,520 & $100 \%$ & 16,889 & $100 \%$ \\
\hline
\end{tabular}

Fuente: SUNAT, BCRP. Elaboración Propia

Cuadro 3

Estructura de la Economía Formal e Informal frente a las exportaciones y empleo

\begin{tabular}{|c|c|c|c|c|c|c|c|}
\hline & & \multicolumn{2}{|c|}{ Cantidad } & \multicolumn{2}{|c|}{ Empleo } & \multicolumn{2}{|c|}{ Exportaciones } \\
\hline & & $\begin{array}{c}\mathrm{N}^{\circ} \text { de } \\
\text { empresas }\end{array}$ & $\%$ & $\begin{array}{c}N^{\circ} \text { de } \\
\text { empleados }\end{array}$ & $\begin{array}{l}\% \text { de la PEA } \\
\text { ocupada }\end{array}$ & $\begin{array}{l}\text { Valor (US\$ } \\
\text { millones) }\end{array}$ & $\%$ \\
\hline $\begin{array}{c}\text { Empresas con } \\
\text { instituciones } \\
\text { para crear } \\
\text { capital }\end{array}$ & $\begin{array}{l}\bar{\varpi} \\
\text { Еँ } \\
\text { एँ }\end{array}$ & 52,133 & $2 \%$ & 626,341 & $10 \%$ & 16,382 & $97 \%$ \\
\hline \multirow{2}{*}{$\begin{array}{c}\text { Empresas sin } \\
\text { instituciones } \\
\text { para crear } \\
\text { capital }\end{array}$} & 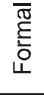 & 439,253 & $17 \%$ & $1,370,945$ & $22 \%$ & 507 & $3 \%$ \\
\hline & 胥 & $2,121,791$ & $81 \%$ & $4,322,234$ & $68 \%$ & $0 \%$ & $0 \%$ \\
\hline \multicolumn{2}{|l|}{ Total } & $2,613,177$ & $100 \%$ & $6,319,520$ & $100 \%$ & 16,889 & $100 \%$ \\
\hline
\end{tabular}

Fuente:ENAHO, 2002. No incluye las empresas agrícolas informales ni las dedicadas al contrabando y al narcotráfico. 
Un hecho adicional es la presencia de actividades como el contrabando y el narcotráfico que, a pesar de ser delictivas, generan impactos dentro de la economía nacional y que representan, según estimaciones realizadas, cerca de $30 \%$ del PBI. Las distorsiones que genera el narcotráfico, por ejemplo, se observan entre otros factores en su impacto en el tipo de cambio, aunado a hechos como las remesas de los peruanos en el exterior y el incremento de las exportaciones de los últimos años.

El Perú por mucho tiempo ha sido y es una economía dolarizada. Los activos y las Reservas Internacionales del BCRP están expresados en la

Gráfico 9

Dolarización de la Economía

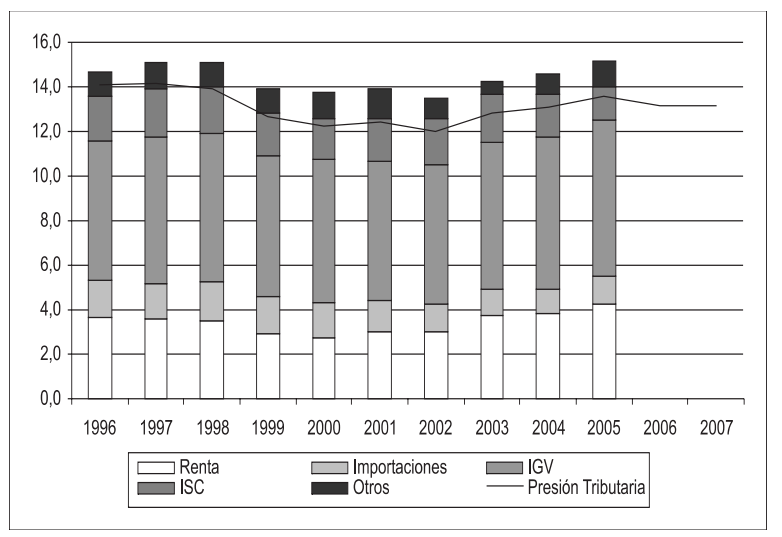

Fuente: BCRP, Nota Semanal № 41 Elaboración Propia divisa norteamericana (US\$). La dolarización de la economía es un factor estructural que define, entre otros, el acceso a la tecnología, la denominación de los ahorros de la población, y los patrones de comercio internacional.

\section{LA OPORTUNIDAD DEL TLC}

Durante el proceso de negociación del TLC se dieron muchas opiniones encontradas acerca de la forma en que el TLC afectará las relaciones comerciales tanto dentro del país como con los vecinos sudamericanos. Pero en las líneas siguientes discutiremos algunos aspectos que se contrapusieron en el debate de si el Perú debe embarcarse en un proceso de complementación comercial tan ambicioso.

Es interesante observar el debate que se planteó entre el Ministerio de Comercio Exterior $^{7}$, encabezado por el entonces ministro Alfredo Ferrero, y CONVEAGRO, uno de los principales gremios que en su propia opinión, se verían afectados con la firma del TLC.

Francke e Iguiñiz, al proponer elementos de viabilidad de las políticas de lucha contra la pobreza, refieren que hasta el momento, en lo que se refiere a políticas de protección del mercado interno frente a la competencia externa, el Perú, para proteger los productos agropecuarios

Cuadro 4

Argumentos sobre el TLC: MITINCI y CONVEAGRO

\begin{tabular}{|c|c|}
\hline $\begin{array}{c}\text { Ministerio de Comercio Exterior y } \\
\text { Turismo }\end{array}$ & Conveagro \\
\hline $\begin{array}{l}\text { Los productos agrarios que se verían } \\
\text { afectados por el TLC Andino son sólo dos, } \\
\text { algodón y trigo, ya que el resto se importa } \\
\text { principalmente desde otros países. }\end{array}$ & $\begin{array}{l}\text { Más del } 80 \% \text { de las importaciones se concentra en trigo, torta de } \\
\text { soya, maíz amarillo, aceite de soya, fibra de algodón y cebada. } \\
\text { EE.UU. sólo es un proveedor importante o dominante en dos de } \\
\text { ellos: trigo y algodón, los cuales además se ubican entre las } 15 \\
\text { principales importaciones. }\end{array}$ \\
\hline $\begin{array}{l}\text { Desgravar totalmente las partidas de } \\
\text { productos que no se importan desde } \\
\text { EE.UU. no tendrá un efecto negativo sobre } \\
\text { nuestra producción. }\end{array}$ & $\begin{array}{l}\text { Se incluyen "cláusulas de preferencia", para que una de las partes } \\
\text { pueda exigir acogerse a las ventajas concedidas de un tercero. } \\
\text { Otros países podrían exigir las condiciones del TLC, denunciando } \\
\text { esta cláusula en sus acuerdos con el Perú. }\end{array}$ \\
\hline $\begin{array}{l}\text { No hay que sobredimensionar el tema de } \\
\text { los subsidios agrarios porque la distorsión } \\
\text { que causan es insignificante. }\end{array}$ & $\begin{array}{l}\text { La importancia de los subsidios no debe ser minimizada. Sólo } \\
\text { recordemos que este tema ha sido una de las principales causas } \\
\text { del fracaso de las sucesivas rondas de la OMC y el mismo ALCA. }\end{array}$ \\
\hline $\begin{array}{l}\text { Los principales reclamos del sector agrario } \\
\text { tienen que ver con la Agenda Interna y no } \\
\text { con el TLC. }\end{array}$ & $\begin{array}{l}\text { Los avances en la Agenda Interna son limitados, el gobierno } \\
\text { asumió compromisos aún no cumplidos (2005) }\end{array}$ \\
\hline $\begin{array}{l}\text { Los daños al productor nacional pueden ser } \\
\text { neutralizados con compensaciones directas. }\end{array}$ & $\begin{array}{l}\text { ¿Es justo que el Estado con graves problemas fiscales, deba } \\
\text { asumir los costos generados por las prácticas de subsidios } \\
\text { realizadas por otros países? Hay problemas de identificación de } \\
\text { los beneficiarios existe filtración y sub-cobertura. }\end{array}$ \\
\hline
\end{tabular}


“... ha descansado sobre todo en las llamadas "franjas de precios", que establecen sobretasas cuando los precios internacionales son demasiado bajos, de tal manera que además protegen de las fluctuaciones del mercado internacional".

Se han usado muy poco otros instrumentos, como cuotas o barreras para arancelarias (como las fitosanitarias). Debido a que en este caso los mecanismos de protección dependen de acuerdos comerciales que implican negociaciones con alcance en múltiples sectores y ámbitos, no puede plantearse sino una política que en estos acuerdos y tratados priorice la defensa de los pequeños productores rurales por su situación de pobreza. Una primera acción necesaria al respecto es la elaboración de estudios sobre los efectos que la reducción de protección a cada producto agropecuario tiene sobre la pobreza rural. En lo inmediato, en relación al TLC con los Estados Unidos y dada la conclusión de las negociaciones, este estudio es aún más ur- gente, así como la discusión de las medidas de compensación necesarias (subsidios directos, etc. $)^{8}$ (El subrayado es nuestro).

Eduardo Morón, investigador de la Universidad del Pacífico y del Instituto Peruano de Economía, manifiesta que:

\section{Gráfico 10:}

Proyección del PBI

$(\mathrm{PBI}=100$ en el año cero - inicio del TLC)

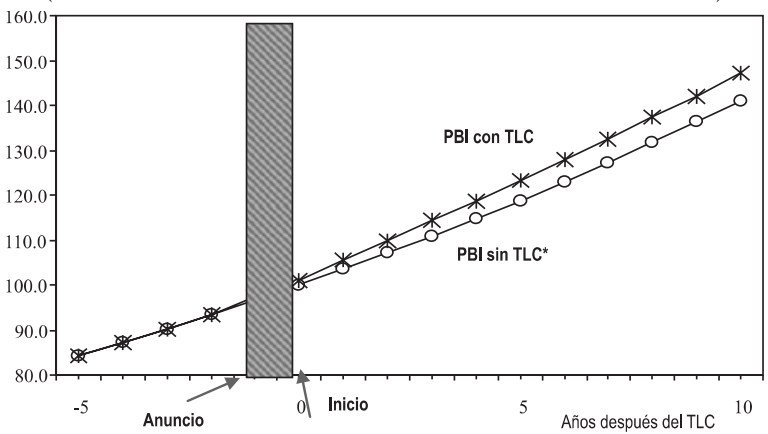

Fuente: Morón, Eduardo (2005)

Gráfico 11:

Esquema de Impactos del TLC PERU - EE. UU.

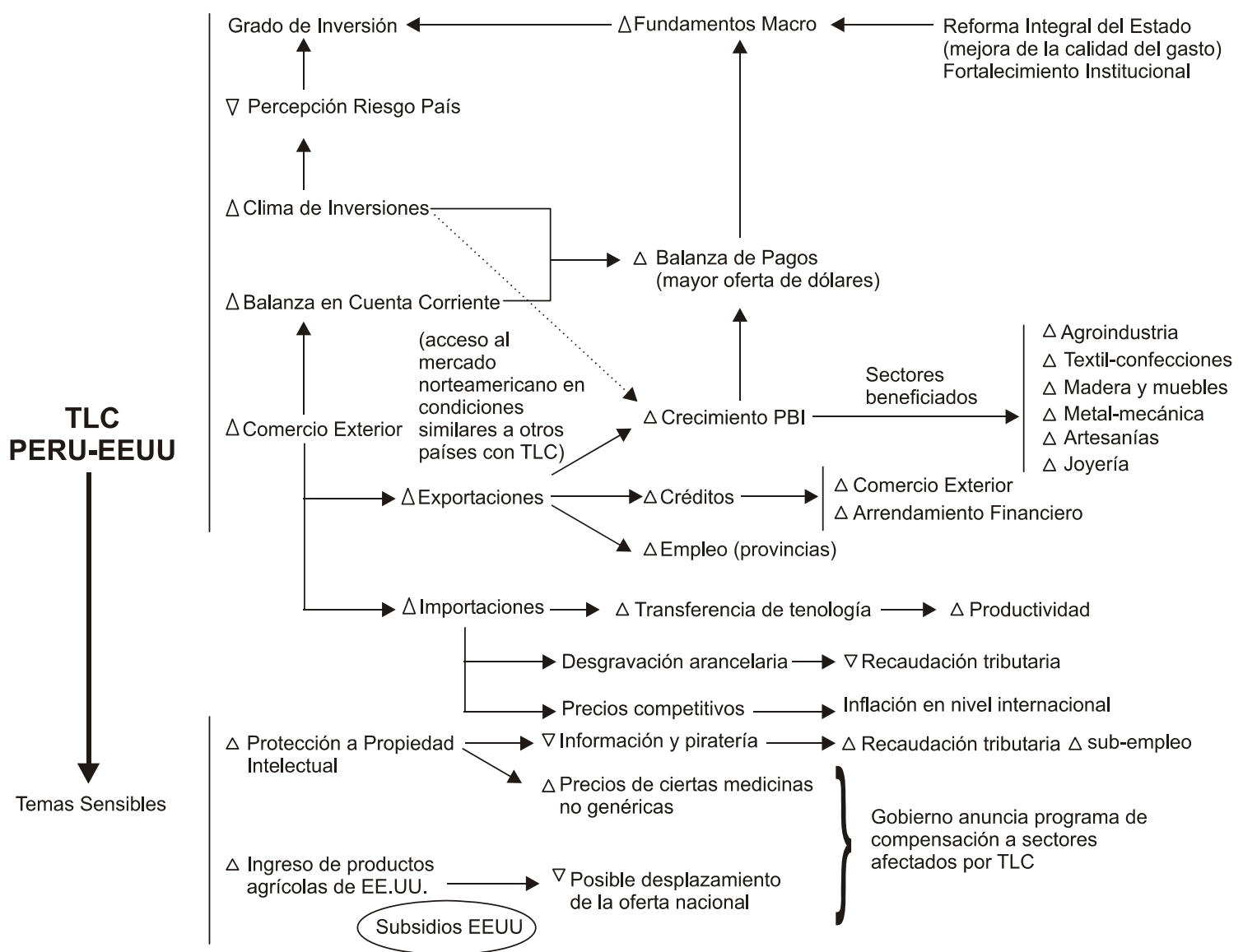

Fuente: Reporte Especial Banco BWS, Diciembre 2005.Elaboración: Departamento de Estudios Económicos del Banco BWS 
“... el impacto que tendría el TLC en la economía peruana sería equivalente a acelerar un punto porcentual a la tasa de crecimiento promedio en — por lo menos - los primeros cinco años posteriores a la firma del acuerdo. Es decir, si nuestra economía crece en promedio 3,5\%, con el TLC con los Estados Unidos creceríamos a un ritmo de 4,5\%. ¿Es esto suficiente? La respuesta definitivamente será que no, pero la idea es justamente adoptar medidas de política que ayuden a que se pueda crecer sosteni-damente, y la firma del TLC con los Estados Unidos es una de ellas, no la única"'. (el subrayado es nuestro).

La investigadora del Centro de Investigación de la Universidad del Pacífico, y actual Ministra de Comercio Exterior, refería que:

"Con el objetivo de revertir esta situación [de no competitividad] el país debe insertarse en el proceso de globalización con una visión de fortalecimiento de la competitividad o productividad (...) enfatizando tres aspectos 1) barreras institucionales, 2) eficiencia de los mercados y 3) la innovación y el desarrollo tecnológico".

En dicho artículo, la autora señala una serie de compromisos que la sociedad, incluido el Estado, debe asumir para promover la iniciativa privada en la generación de competitividad, que son: fortalecimiento institucional, transparencia en la gestión, eliminación de las restricciones a la eficiencia de los mercados, políticas de apertura, etc. Como alternativas señala la reducción de barreras burocráticas y el fortalecimiento de las entidades promotoras de supervisión de la competencia ${ }^{10}$.

Como hemos indicado al inicio del presente artículo, se trata de identificar estas características estructurales que hacen que el impacto de un TLC no sea aprovechado por todos sino que a diferencia de otras latitudes, no se incorporen los progresos tecnológicos sino por el contrario, se acentúe la ya marcada brecha entre demanda interna y producción para exportación.

En el reporte preparado por el Departamento de Estudios Económicos del Banco Wiese Sudameris (BWS), se refiere lo que se ha propagado a través de los medios de comunicación, sobre la importancia del TLC en la economía peruana, y resume sus apreciaciones en el siguiente Gráfico (Ver Gráfico 11).

Es notable señalar que indica como premisas para la interacción del TLC con el Perú tanto la reforma integral del Estado y el fortalecimiento institucional, como la compensación a los afectados en lo temas sensibles; además el cuadro mostrado ejemplifica el impacto de un aumento de las importaciones que implica impactos en transferencia de tecnología y, por ende, en la productividad.

Por último haremos referencia al trabajo del Profesor Tello quien compiló diferentes estudios

\section{Cuadro 5}

Impactos de sobre Exportaciones, Importaciones y PBI

\begin{tabular}{|l|c|c|c|c|c|c|c|}
\hline \multicolumn{1}{|c|}{ Estudio } & \multicolumn{5}{|c|}{ Estados Unidos, 2002 } & \multicolumn{3}{c|}{ Perú, 2002 - 2004 } & $\begin{array}{c}\text { 年ecto sobre la tasa de } \\
\text { crecimiento del PBI } \\
\text { real del Perú }\end{array}$ \\
\hline & $\% \mathrm{X}$ & $\% \mathrm{M}$ & $\% \mathrm{PBI}$ & $\% \mathrm{X}$ & $\% \mathrm{M}$ & $\% \mathrm{PBI}$ & \\
\hline $\begin{array}{l}\text { Fairle (2005) y } \\
\text { Fairle Cuadra y } \\
\text { Floran (2004) }\end{array}$ & 0,23 & 0,17 & 0,02 & 25,6 & 19,2 & 3,4 & $0,43-7 \%$ \\
\hline Tello (2005, 2004) & 0,23 & 0,17 & 0,02 & 25,6 & 19,2 & 3,4 & $0,28 \%$ \\
\hline $\begin{array}{l}\text { Rodriguez, } \\
\text { Secunario, Ascorne y } \\
\text { Molina (2004) }\end{array}$ & 0,23 & 0,17 & 0,02 & 25,6 & 19,2 & 3,4 & $-2,24 \%$ \\
\hline $\begin{array}{l}\text { Morón, Bernedo, } \\
\text { Chavez, Cusaco y } \\
\text { Wiokelned (2004) }\end{array}$ & 0,23 & 0,17 & 0,02 & 25,6 & 19,2 & 3,4 & $0,527-3,285 \%$ \\
\hline $\begin{array}{l}\text { Ministerio de } \\
\text { Comercio Exterior y } \\
\text { Turismo (2005) }\end{array}$ & 0,23 & 0,17 & 0,02 & 25,6 & 19,2 & 3,4 & $2,31 \%$ \\
\hline
\end{tabular}

Fuente: Tello (2005) 
sobre los impactos de la desgravación en Perú y Estados Unidos, que se resume en el Cuadro 5, donde además refiere que:

“...consistentes con los resultados de estudios similares que los analizados en esta sección, todos los estudios - menos uno- estiman que la desgravación arancelaria propuesta en el TLC Perú-Estados Unidos tiene impactos económicos globales positivos o beneficios netos positivos, aunque de distinta magnitud. Los beneficios positivos provenientes del primer tipo de efectos, el de nivel, y originados por la desgravación completa, en general no son significativos. Los beneficios netos estimados son de un aumento del PBI real menor de $1 \%$. Estos bajos beneficios se explican por las bajas tasas promedio arancelarias iniciales que Estados Unidos impone actualmente al Perú y el hecho de que dichas tasas estén concentradas en un conjunto pequeño - con respecto al universo arancelario- de partidas -en particular aranceles concentrados en los sectores textiles, vestidos y ciertos productos agrícolas"11.

\section{HACIA UN TLC INTERNO}

¿Qué actitud ha tomado el actual gobierno con respecto al TLC? En el mes de septiembre del 2006, durante su presentación ante el Congreso, el actual Presidente del Consejo de Ministros dijo:

“... queremos resaltar la necesidad que el TLC no beneficie sólo a una minoría de empresas peruanas (2\% de empresas que emplea al $10 \%$ de la PEA), sino a un mayor número de empresas y de peruanos que deben ser los beneficiarios directos o indirectos de este importante Tratado. Es decir, tenemos que trabajar la agenda al interior de nuestro país, para que las oportunidades de mayores exportaciones alcancen a nuestros agricultores e industriales y eso signifique miles de nuevos puestos de trabajo. Obviamente no puedo dejar de resaltar los beneficios laborales que trae este TLC, dicho claramente, sólo podrán exportar las empresas que cumplan con los derechos de sus trabajadores y con todas las reglas establecidas por la Organización Internacional del Trabajo (OIT), es decir tendremos más puestos de trabajo, pero trabajo digno con pleno respeto de los derechos de nuestros trabajadores" 12 .

Es decir, el Estado peruano reconoce la figura del acuerdo comercial como una oportunidad de aprovechar los beneficios del comercio internacional con un país como Estados Unidos, pero también reconoce que es necesario que éste llegue a un mayor número de personas.

Planteamos las siguientes interrogantes:

- ¿Es posible desligar las consecuencias económicas de las sociales, tras la firma del Acuerdo de Complementación Económica, hoy a la espera de la aprobación del Congreso Norteamericano?

- ¿El patrón de comercio en los Estados Unidos tiene por interés ampliar el comercio de granos u otros productos agrícolas con el Perú, o es más importante en otros rubros, como la investigación científica, producción de tecnología y protección de patentes? En cambio el Perú, ¿no está regresando a patrones de comercio dentro de la división internacional del trabajo, en que nos convertimos en proveedor de bienes intermedios y no de bienes finales?

- ¿Se ha determinado si el impacto en el empleo está a la par con el impacto en la producción y en los ingresos por exportaciones? Este hecho se agrava al tener el Perú una informalidad que supera la lentitud del sistema legal.

- ¿La incorporación de un plan de compensaciones no forma parte de una solución no estructural del problema? ¿Cómo orientar la competitividad del país, sin perjudicar a los actores más vulnerables de la sociedad?

- Los esfuerzos de cambio estructural desde una legalidad que desalienta la incorporación de empresas y empleos, deben enfrentar múltiples circunstancias y problemas, como por ejemplo, la incorporación de los beneficios de las políticas comerciales a la mayorías de la población.

\section{NOTAS}

1 De Soto, Hernando (1990). El otro sendero. Octava Edición. Bogotá, Instituto Libertad y Democracia.

2 Abusada Salah, Roberto; Taboada, Saray; y Chávez, Jorge F. (2004) Impactos sectoriales y regionales de la Ley de Preferencias Arancelarias y de Erradicación de Drogas 
(ATPDEA). Lima, Proyecto Crecer y USAID, Septiembre 2004.

3 Encuesta a Nivel Nacional de la Empresa Apoyo S.A. publicada por el Diario El Comercio. 12 de mayo del 2006.

4 Seminario de Marzi, Bruno y Molina Campodónico, Oscar. Efectos del TLC sobre el empleo. Por encargo del Programa de las Naciones Unidas para el Desarrollo PNUD y el Ministerio de Trabajo. Septiembre 2004.

5 En Bustelo, Pablo (1998). Teorías Contemporáneas del desarrollo económico. Serie Historia del Pensamiento Económico. Madrid, Editorial Síntesis.

6 López Mas, Julio. (2003) Globalización y Economías regionales del Perú. En Gestión en el Tercer Milenio, Revista de Investigación de la Facultad de Ciencias Administrativas, UNMSM. Año 6, № 11. Julio 2003.

7 Ministerio de Comercio Exterior y Turismo (2005). Análisis del impacto del Tratado de Libre Comercio Perú-EE. UU. Lima, Ministerio de Comercio Exterior y Turismo.

8 Francke, Pedro e Iguiñiz, Javier. (2005) Crecimiento con Inclusión en el Perú. Documento de Trabajo. Agencia de Cooperación Suiza para el Desarrollo, Octubre del 2005.

9 Morón, Eduardo y otros. (2005). Tratado de Libre Comercio con los Estados Unidos: una oportunidad para crecer sostenidamente. Primera Edición. Universidad del Pacífico, CIUP, Instituto Peruano de Economía.

${ }^{10}$ Araoz, Mercedes (2006). Apuntes para una agenda interna de competitividad frente a la globalización. En Economía y Sociedad, Revista de Investigación del Consorcio de Investigación Económico y Social (CIES), № 59, Marzo 2006.

11 Tello, Marco. (2006) Marco técnico sobre el impacto económico del TLC Perú- EE. UU., aparecido en la Página Web del Consorcio de Investigación Económica y Social CIES. Lima, 2006.

${ }^{12}$ Exposición del Presidente del Consejo de Ministros Dr. Jorge del Castillo Gálvez ante el Congreso de la República. Septiembre del 2006.

\section{BIBLIOGRAFIA}

Abusada Salah, Roberto; Taboada, Saray, y Chávez, Jorge F. (2006). Impactos sectoriales y regionales de la Ley de Preferencias Arancelarias y de Erradicación de Drogas (ATPDEA). Proyecto Crecer y USAID. Lima, Septiembre 2004.

Araoz, Mercedes, (2006). Apuntes para una agenda interna de competitividad frente a la globalización. En Economía y Sociedad. Revista de Investigación del Consorcio de Investigación Económico y Social, CIES, № 59, Marzo del 2006.

Banco Central de Reserva del Perú. (2006). Memoria Anual 2005. Lima.

Banco Central de Reserva del Perú (2006). Cuadros Estadísticos de la Nota Semanal. $N^{o}$ 39, 40, 41 y 42. Lima.
Bustelo, Pablo. (1998). Teorías Contemporáneas del desarrollo económico. Serie Historia del Pensamiento Económico. Madrid, Editorial Síntesis.

De Soto, Hernando. (1990). El otro sendero. Octava Edición. Bogotá, Instituto Libertad y Democracia.

Eduardo Morón y otros. (2005). Tratado de Libre Comercio con los Estados Unidos: una oportunidad para crecer sostenidamente. Primera edición. Universidad del Pacífico, CIUP, Instituto Peruano de Economía. Diciembre 2005 .

Francke, Pedro e Iguiñiz, Javier. (2005). Crecimiento con Inclusión en el Perú, Documento de Trabajo. Agencia de Cooperación Suiza para el Desarrollo. Octubre 2005.

Instituto Nacional de Estadística e Informática (INEI). (2006). Informe Económico Mensual: PERÚ. Informe Mensual de Marzo del 2006. Informe preparado por la Dirección Técnica de Indicadores Económicos, Lima, Mayo 2006.

López Mas, Julio. (2003). Globalización y Economías regionales del Perú. En Gestión en el Tercer Milenio, Revista de Investigación de la Facultad de Ciencias Administrativas, UNMSM. Año 6, № 11, Julio 2003.

Ministerio de Comercio Exterior y Turismo. (2005). Análisis del impacto del Tratado de Libre Comercio Perú-EE. UU. Lima, Ministerio de Comercio Exterior y Turismo.

Marzi, Bruno y Molina Campodónico, Oscar. Seminario Efectos del TLC sobre el empleo. Por encargo del Programa de las Naciones Unidas para el Desarrollo PNUD y el Ministerio de Trabajo. Septiembre 2004.

Tello, Marco. (2006). Marco Técnico sobre el impacto económico del TLC Perú - EE. UU., aparecido en la Página Web del Consorcio de Investigación Económica y Social, CIES. Lima, 2006.

http://www.pucp.edu.pe/economia/ TLCendebate.pdf

http://cies.org.pe/es/TLC

http://www.bcrp.gob.pe/

http://www.inei.gob.pe/publicaciones/ 